\title{
THE INFLUENCE OF ENVIRONMENTAL FACTORS ON THE ONSET OF RHEUMATOID ARTHRITIS
}

\author{
Cristina Suta $^{1,2}$, Sabina Ciocodei $^{2}$, Iulia Andronache ${ }^{2}$, Elvira Craiu ${ }^{1}$, Maria Suta ${ }^{1,2}$ \\ ${ }^{1} 3^{\text {rd }}$ Department, $I^{\text {st }}$ Clinical Disciplines, Faculty of Medicine, Ovidius University, Constanta \\ ${ }^{2}$ Rheumatology Department, $2^{\text {nd }}$ Internal Medicine Clinic, Sf. Apostol Andrei Emergency Clinical County \\ Hospital, Constanta
}

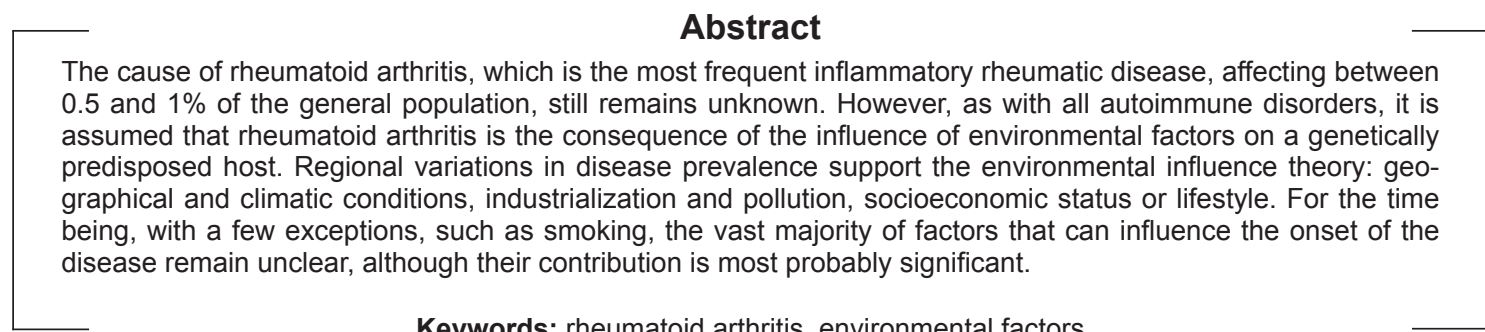

Keywords: rheumatoid arthritis, environmental factors

\section{INTRODUCTION}

The presence of the disease in approximately only $15 \%$ of genetically identical twins outlines the influence of infectious or non-infectious factors on the development of rheumatoid arthritis (RA).

Epidemiological studies have shown differences in what regards the prevalence of the disease amongst the same type of population, according to rural or urban environment, geo-climactic conditions, exposure to ultraviolet radiation, industrialisation and pollution, endemic infectious and lifestyle, such as diet and smoking. The analysis of RA epidemiology can contribute to the identification of environmental factors involved in the onset of the disease in a restricted genetic context.

\section{Smoking}

Smoking is the best studied environmental risk factor involved in the development of RA. In a metaanalysis which identified 16 studies published in Medline and EMBASE (between 1996 and December 2006) that evaluated the association between smoking and disease onset risk, smoking was proven as a risk factor in heavy smokers (more than 20 pack years) and for seropositive disease $(\mathrm{RF}+)$ in men (1). The study of genetic and environmental factors shows that the influence of the environment, lifestyle habits such as smoking - or other randomly intervening factors, also plays an essential role in the appearance of antiCCP antibodies and RA with positive antiCCP (2). Smoking and current smoking status has been proven as an independent risk factor for the radiological progression in all cases of early RA (3).

Smokers, and in particular heavy smokers, have an increased risk of developing RA even twenty years after smoking cessation (4). The association between smoking status and shared epitope containing HLA genes (HLA-DRB1 alleles) significantly increases the risk for antiCCP-positive disease both in Caucasians (OR between 20-25 for the association HLA-DRB1 and smoking) (5) and in Asians (6).

\section{Other environmental factors}

Numerous other risk factors apart from smoking, such as socio-economic status and level of education, exposure to silica dust, polluted atmosphere, exogenous hormone administration, infections, periodontitis, exposure to ultraviolet radiation, vitamin D plasmatic levels, and alcohol consumption can modify the risk for disease development (7). How-

Correspondence address:

Cristina Suta, MD, Sf. Apostol Andrei Emergency Clinical County Hospital, 145 Tomis Blvd, 900591, Constanta, Romania

E-mail: cris_duminica@yahoo.com 
ever, for the time being, with the exception of smoking, the vast majority of risk factors remain unidentified or lacking sufficient data, even though their contribution to disease onset is probably significant.

\section{Occupational environmental factors}

Exposure to silica dust, mineral oils or organic solvents represent types of professional exposure with a proven influence on the risk of developing RA.

The relationship between silica dust exposure and RA has been intensively studied. A meta-analysis published in 2002 (8) presents the results of 10 epidemiological studies between 1986 and 2001, which totalled 242 cases of RA associated with silica exposure. Nine of the ten studies included in this meta-analysis outline the increased risk for disease development after exposure to silica dust from various sources; in particular for men, a threefold increase in the global relative risk $(3.43,95 \% \mathrm{Cl} 2.25-5.22)$ compared to patients for RA control lots. The results of the first part of the EIRA study ("epidemiological investigation of rheumatoid arthritis"), a populational case-control study which included over 900 RA patients, ages 18 to 70, residing in a geographical areas in central and southern Sweden, outline a threefold increase in disease onset risk (OR: 1.2 to 7.6 ) in male patients with significant professional exposure to silica dust (9). The second part of the EIRA study reports that male patients, active smokers and exposed to silica, have a 7.4 times higher risk of developing RA with positive antiCCP compared to non-smokers without exposure to silica (10).

Professional exposure to mineral oils and the risk of developing RA represents another objective of the EIRA study: men with either respiratory or cutaneous professional exposure to mineral oils have an increased risk of developing seropositive $\mathrm{RA}$ : $\mathrm{RR}=1.4$ for RA (RF+) and 1.6 for RA antiCCP (+) (11).

An increased risk for developing $\mathrm{RA}$ has also been reported in professions that involved significant inhalation of organic solvents (upholsterer, hairdresser and blacksmith-bender) (12). Hairdressers, for example, have a threefold increased risk for developing RA (OR 3.0; 95\% Cl 1.0-9.4) compared to non-exposed women (13).

\section{Mediterranean diet}

The Cretan diet, with fruit, vegetables, cereal, fish, olive oil, less red meat and alcohol in moderate quantities $(\leq 8 \mathrm{~g} /$ day $)$ is the prototype of the Mediterranean diet, considered as being the healthiest type of diet. Several case-control studies have proven the beneficial effect of the Mediterranean diet on RA disease risk and evolution. A prospective study conducted in a certain geographic area of Sweden, between 1996 and 2005, which included 1889 incident cases of RA and 2145 control cases, outlined a moderate decrease of disease development risk in those who consumed fish (1-7 days/week) and fish oil (1-3 times/month) (14).

The most recent data regarding the association between RA incident cases and the Mediterranean diet come from the analysis of the NHS I (Nurses' Health Study) and NHS II studies. The studies were conducted between 1980 and 2008 and 1991 and 2009 respectively, and they prospectively followed 83.245 participants for NHS I and 91.393 for NHS II. The analysis, adjusted according to other risk factors, did not evidence a decrease in disease risk for those who adopted the Mediterranean diet (15). However, the multivariate analysis of RA incident case from these two studies did outline an increase in the risk of seropositive RA in association with regular consumption of carbonated drinks (including regular coca-cola, decaf coca-cola and other sweet carbonated drinks): consumption of $\geq 1$ carbonated drink determined a $63 \%$ increase (HR: $1.63 ; 95 \mathrm{Cl}$ : $1.15,2.30$, P-trend $=0.004$ ) in the risk of seropositive RA onset compared to those who drank none or one sweet carbonated drink at most per month. The analysis of a sub-sample of NHS patients with late onset-RA (age at onset $>55$ years) showed a stronger association with carbonated drink consumption (HR: 2.64; 95\% Cl: 1.56, 4.46; P-trend $<0.0001$ ) (16).

\section{Alcohol consumption}

In literature on the matter, several clinical casecontrol studies show a decreased risk for RA in people who consume alcohol. Data from two case-contol studies conducted in the Nordic countries in Europe - the Swedish EIRA study and the Danish CACORA study - outline a 5\% risk reduction (EIRA) and 2\% respectively (CACORA) with the consumption of $16 \mathrm{~g}$ of alcohol (one drink), an inversely proportional relationship for disease risk with dose of consumed alcohol and a attenuation of the effect of the two established risk factors (shared epitope HLA-DRB1 alleles and smoking) (17).

The protective effect of alcohol consumption and the inversely proportional relationship between the amount of consumed alcohol and disease risk are 
also supported by a case-control study which included 873 RA patients and 1004 healthy individuals in the control group. All patients, recruited from the rheumatology department of a Sheffield hospital (Royal Halamshire Hospital) between 1999 and 2006, with RA according to the 1987 ACR criteria, disease duration of at least 3 years and at least one erosion on hands or feet X-ray. Regular alcohol consumption (more than 10 days a month) decreases the risk for RA 4.17 times (3.01-5.77) compared to nondrinkers (18).

Results of a recent metaanalysis support the protective effect of low and moderate alcohol doses. This effect begins to lose its significance if daily consumed doses exceed $15 \mathrm{~g}$ /day (19). Analysis of the data from two other prospective studies regarding RA risk factors, NSH I and NHS II, show a modest association between moderate alcohol consumption and the risk of RA development: consumption of 5.0-9.9 g/day (the equivalent for 3-5 standard drinks per week) leads to a $22 \%$ decrease in the risk for RA development and $31 \%$ decrease in the risk for seropositive RA development compared to women who do not consume alcohol. The analysis of the type of drink shows that beer consumption and not wine or hard drinks is associated with risk reduction (20).

\section{Exposure to ultraviolet radiation}

Recent data regarding the risk of developing RA and exposure to ultraviolet radiation comes from the analysis of the two large prospective studies, NHS I and NHS II, which followed risk factors associated with new cases of RA amongst a large population sample of women (106.368 women in NHS I and 115.561 women in NHS II) with ages between 30 and 55 years old in 1976 and 25-42 years old in 1989 respectively, which were followed until 2008 (NSH I) and 2009 (NHS II). Cumulative exposure to UV-B flux (low, average, high) represents a composite appreciation of ambient exposure to UV according to latitude, altitude, and degree of cloudiness of the state of residence. In NHS I, high exposure to UV-B was correlated with a decreased risk of RA of up to $21 \%$, but this correlation was not registered in NHS, probably due to the use of sunscreen cream by the younger generation (21).

\section{Vitamin D}

Data regarding the relationship between vitamin and the risk of RA development are contradictory.
While the Iowa Women's Health Study, which prospectively followed a sample population of 29.368 women aged 55 to 69 years old for 11 years, showed a decrease disease risk in women who ingested high doses of vitamin D (22), other studies do not observe a significant correlation between serum levels of 25 $\mathrm{OH}$ vitamin $\mathrm{D}$ and the risk of developing RA $(23,24)$.

\section{Atmospheric pollution}

In recent years, atmospheric pollution has been considered as a potential risk factor for RA. Traffic is considered a surrogate for atmospheric pollution, even though numerous other air pollution sources exist: fuel burning industry, forest fires, as well as solid fuel burning.

The association between atmospheric pollution and RA has been investigated in three major studies: Nurses' Health Study (NHS) in the SUA (it included 90.927 women) $(25,26)$, British Colombian (BC) Study in Canada (640.041 subjects were included) (27) and the Swedish Epidemiological Investigation of Rheumatoid Arthritis (EIRA) (1.497 new cases of RA and 2536 control cases) (28), with rather different results.

Two out of the three studies, NSH and EIRA, have calculated the risk for RA through exposure to atmospheric pollution, adjusting results after exclusion of other risk factors, such as smoking and socioeconomic status. In NHS, RA incidence according to the distance from the residence to the highway was studied. Multivariate analysis, which excluded other potential risk factors (age, race, smoking, BMI, and so on), outlined the fact that women who lived 50 meters at most from the roadways had a higher risk of developing RA compared to those who lived at least 200 meters away $(\mathrm{HR}=1.31 ; 95 \% \mathrm{Cl} 0.98$ 1.74). Differences were more evident in the case of non-smoking women $(\mathrm{HR}=1.62 ; 95 \% \mathrm{Cl}, 1.04-2.52)$ (25).

The EIRA study followed the risk of RA onset in relation to atmospheric pollution by measuring the degree of pollution caused by traffic and home heating sources 5, 10 and 20 years before the first symptoms of the disease. An increase in global RA risk was registered in relation to exposure to pollutant gases $\left(\mathrm{NO}_{2}\right.$ and $\left.\mathrm{SO}_{2}\right) 10$ years before onset, but after adjusting results according to smoking and education level, the significance of the results was reduced $(\mathrm{OR}=1.18,95 \%, \mathrm{Cl} 0.97-1.43$ and $\mathrm{OR}=1.09,95 \%$, Cl: $0.99-1.19$ for $\mathrm{SO}_{2}$ and $\mathrm{NO}_{2}$ respectively). This 
stratified analysis outlined a maintained level of risk for antiCCP-negative RA even after smoking and education adjustments $(\mathrm{OR}=1.48,95 \% \mathrm{Cl} 1.13$ 1.95 and $\mathrm{OR}=1.22,95 \% \mathrm{Cl} 1.07-1.40$ for $\mathrm{SO}_{2}$ and $\mathrm{NO}_{2}$ respectively) (28).

Atmospheric pollution was not associated with antibody positivity or RA signs neither in populations with high risk (first degree relatives of RA patients), followed in a prospective study began in 2002 up till 2012, which included 1.767 participants from Colorado (39\%), California (29\%), Nebraska (13\%), Washington (11\%), and New York (8\%). RA risk in relation to pollution was calculated after adjustments made for age, ethnicity, gender, active smoking, and level of education (29).

\section{Socio-economic environment}

The EIRA study, which included newly diagnosed cases of RA between 1996 and 2001 from a given geographic area of Sweden, also analysed socio-economic status (level of education, occupational class - both in accordance with Swedish classifying systems) as a risk factor for the disease. In this study, 930 cases of RA were included (654 women and 276 men, with a mean disease duration of 10 months) and 1.357 control-cases. The lowest disease risk was registered amongst subjects with higher education and the lowest degree of manual activity. The effect of socio-economic status was more evident in seropositive RA cases (30).

\section{Postmenopausal hormonal therapy}

RA is an autoimmune disease with a evident preference for the female gender, regardless of age, but the highest incidence is registered for women ages 55 to 64 years old, in peri- and postmenopausal periods (31), possibly due to the influence of hormonal factors, such as oestrogen (32).

The relationship between hormone in use in postmenopause and the risk for RA was explored in numerous studies that offered contradictory data. In
2015, the results of the EIRA study were published, which, for the first, investigated the association between the use of postmenopausal hormone therapy and the risk for developing antiCCP seropositive RA compared to antiCCP-negative RA, concluding that hormonal therapy in postmenopause can reduce the risk of seropositive RA in women over $>50$ years old, who were actively using hormonal therapy at disease onset, but that there is no correlation in what regards seronegative RA (33).

\section{Other potential risk factors}

Studies published in the last years have shown correlations with other potential risk factors, such as:

- A diet high in sodium increases the risk of seropositive RA in smokers (34) (there is recent data offered by studies performed on animals regarding the importance of sodium in the development of autoimmune diseases through Th17 cells) (35);

- Gastrointestinal and urogenital infections are associated with a decreases risk of RA, hypothetically through alterations of intestinal microbial flora (36).

- Physical activity can be a protective factor in RA aetiology (37).

A more profound analysis of RA epidemiology may contribute to identification of environmental factors that influence the onset of the disease in a restricted genetic context. For the time being, with a few exceptions, such as smoking, the role of other environmental factors, although significant, remains a good investigation subject. The relation between genetic predisposition and environment influence is unclear, one of the modalities of intervention in the onset of the disease might be the epigenetic changes, influencing both its course and the response to specific treatment. Thus, epigenetic changes induced by environmental factors represent one of the future research topics regarding etiopathogeny of rheumatoid arthritis (38).

\section{REFERENCES}

1. Sugiyama D. et al. Impact of smoking as a risk factor for developing rheumatoid arthritis: a meta-analysis of observational studies. Ann Rheum Dis. 2010 Jan; 69(1):70-81.

2. Aase Haj Hensvold et al. Environmental and genetic factors in the development of anticitrullinated protein antibodies (ACPAs) and ACPA-positive rheumatoid arthritis: an epidemiological investigation in twins. Ann Rheum Dis 2015; 74:375-380.

3. Saedis Saevarsdottir et al. Current smoking status is a strong predictor of radiographic progression in early rheumatoid arthritis: results from the SWEFOT trial. Ann Rheum Dis 2015; 74:1509-1514.

4. Costenbader K.H., Feskanich D., Mandl L.A. et al. Smoking intensity, duration, and cessation, and the risk of rheumatoid arthritis in women. Am J Med. 2006 Jun; 119(6):503-9. 
5. Kallberg H., Padyukov L., Plenge R.M., et al. Gene-gene and gene-environment interactions involving HLA-DRB1, PTPN22, and smoking in two subsets of rheumatoid arthritis. Am J Hum Genet. 2007 May; 80(5):867. -75.

6. Bang S.Y., Lee K.H., Cho S.K. et al. Smoking increases rheumatoid arthritis susceptibility in individuals carrying the HLADRB1 shared epitope, regardless of rheumatoid factor or anti-cyclic citrullinated peptide antibody status. Arthritis Rheum. 2010 Feb; 62(2):369-77.

7. Karlson E.W., Deane K. Environmental and gene-environment interactions and risk of rheumatoid arthritis. Rheum Dis Clin North Am. 2012 May; 38(2):405-26.

8. Khuder S.A., Peshimam A.Z., Agraharam S. et al. Environmental risk factors for rheumatoid arthritis. Rev Environ Health. 2002 Oct; 17(4):307-15.

9. Stolt P., Kallberg H., Lundberg I. et al. Silica exposure is associated with increased risk of developing rheumatoid arthritis: results from the Swedish EIRA study. Ann Rheum Dis. 2005 Apr; 64(4):582-6).

10. Stolt P., Yahya A., Bengtsson C. et al. Silica exposure among male current smokers is associated with a high risk of developing ACPA-positive rheumatoid arthritis. Ann Rheum Dis. 2010 Jun; 69(6):1072-6).

11. Sverdrup B., Kallberg H., Bengtsson C., et al. Association between occupational exposure to mineral oil and rheumatoid arthritis: results from the Swedish EIRA case-control study. Arthritis research \& therapy. 2005; 7:R1296-R1303.

12. Olsson A.R., Skogh T., Axelson O. et al. Occupations and exposures in the work environment as determinants for rheumatoid arthritis. Occup Environ Med. 2004 Mar; 61(3):233-8.

13. Olsson A.R., Skogh T., Wingren G. Occupational determinants for rheumatoid arthritis. Scand J Work Environ Health. 2000 Jun; 26(3):243-9.

14. Rosell M., Wesley A.M., Rydin K. et al. Dietary fish and fish oil and the risk of rheumatoid arthritis Epidemiology: November 2009 Volume 20 - Issue 6 - pp 896-901.

15. Hu Y., Costenbader K.H., Gao X. et al. Mediterranean diet and incidence of rheumatoid arthritis in women. Arthritis Care Res (Hoboken). 2015 May; 67(5):597-606.

16. Hu Y., Costenbader K.H., Gao X. et al. Sugar-sweetened soda consumption and risk of developing rheumatoid arthritis in women. Am J Clin Nutr. 2014 Sep; 100(3):959-67.

17. Källberg H., Jacobsen S., Bengtsson C. et al. Alcohol consumption is associated with decreased risk of rheumatoid arthritis: results from two Scandinavian case-control studies. Ann Rheum Dis. 2009 Feb;68(2):222-7.

18. Maxwell J.R., Gowers I.R., Moore D.J. et al. Alcohol consumption is inversely associated with risk and severity of rheumatoid arthritis. Rheumatology (Oxford). 2010 Nov; 49(11):2140-6.

19. Zhichao Jin, Chun Xiang, Qing Cai et al. Alcohol consumption as a preventive factor for developing rheumatoid arthritis: a doseresponse meta-analysis of prospective studies. Ann Rheum Dis 2014; 73:1962-1967.

20. Bing Lu, Solomon D., Costenbader K.H, et al. Alcohol Consumption and Risk of Incident Rheumatoid Arthritis in Women: a Prospective Study. Arthritis Rheumatol. 2014 August; 66(8): 1998-2005.

21. Arkema E.V., Hart J.E., Bertrand K.A. et al. Exposure to ultraviolet-B and risk of developing rheumatoid arthritis among women in the Nurses' Health Study. Ann Rheum Dis. 2013 Apr; 72(4):506-11. doi: 10.1136/annrheumdis-2012-202302. Epub 2013 Feb 4.
22. Merlino L.A., Curtis J., Mikuls T.R. et al. Vitamin D intake is inversely associated with rheumatoid arthritis: results from the lowa Women's Health Study. Arthritis and rheumatism. 2004; 50:72-77).

23. Feser M., Derber L.A., Deane K.D. et al. Plasma $25, \mathrm{OH}$ vitamin $D$ concentrations are not associated with rheumatoid arthritis (RA)related autoantibodies in individuals at elevated risk for RA. J Rheumatol. 2009; 36:943-946.

24. Hiraki L.T., Arkema E.V., Cui J., Malspeis S. et al. Circulating 25-hydroxyvitamin $D$ level and risk of developing rheumatoid arthritis. Rheumatology (Oxford). 2014 Dec; 53(12):2243-8.

25. Hart J.E., Laden F., Puett R.C. et al. Exposure to traffic pollution and increased risk of rheumatoid arthritis. Environ Health Perspect. 2009; 117:1065-9.

26. Hart J.E., Kalberg H., Laden F. et al. Ambient air pollution exposures and risk of rheumatoid arthritis in the Nurses' Health Study. Arthritis Care Res (Hoboken). 2013; 65(7):1190-6.

27. De Roos A.J., Koehoorn M., Tamburic L. et al. Proximity to traffic, ambient air pollution, and community noise in relation to incident rheumatoid arthritis. Environ Health Perspect. 2014; 122:1075-80.

28. Hart J.E., Källberg H., Laden F. et al. Ambient air pollution exposures and risk of rheumatoid arthritis: results from the Swedish EIRA case-control study. Ann Rheum Dis. 2013; 72(6):888-94)

29. Gan R.W., Deane K.D., Zerbe G.O. et al. Relationship between air pollution and positivity of RA-related autoantibodies in individuals without established RA: a report on SERA. Ann Rheum Dis. 2013; 72(12):2002-5.

30. Bengtsson C., Nordmark B., Klareskog L. et al. on behalf of the EIRA study Group. Socio-economic status and the risk of developing rheumatoid arthritis: results from the Swedish EIRA study. Ann Rheum Dis 2005; 64:1588-1594.

31. Humphreys J.H., Verstappen S.M., Hyrich K.L. et al. The incidence of rheumatoid arthritis in the UK: comparisons using the 2010 ACR/EULAR classification criteria and the 1987 ACR classification criteria. Results from the norfolk arthritis register. Ann Rheum Dis. 2013; 72(8):1315-20.

32. Doran M.F., Crowson C.S., O'Fallon W.M. et al. The effect of oral contraceptives and estrogen replacement therapy on the risk of rheumatoid arthritis: a population based study. J Rheumatol. 2004; 31(2):207-13

33. Cecilia Orellana et al. Postmenopausal hormone therapy and the risk of rheumatoid arthritis: results from the Swedish EIRA population-based case-control study. Eur J Epidemiol (2015) 30:449-457.

34. Bjorn Sundstrom et al. Interaction between dietary sodium and smoking increases the risk for rheumatoid arthritis: results from a nested case control study. Rheumatology 2015; 54:487493.

35. Kleinewietfeld M., Manzel A., Titze J. et al. Sodium chloride drives autoimmune disease by the induction of pathogenic T17 cells. Nature 2013; 496:51822).

36. Maria E., Sandberg C. et al. Recent infections are associated with decreased risk of rheumatoid arthritis: a population-based casecontrol study. Ann Rheum Dis 2015; 74:904-907.

37. Daniela Di Giuseppe et al. Physical activity and risk of rheumatoid arthritis in women: a population-based prospective study. Arthritis Research \& Therapy (2015) 17:40.

38. Shichikawa K., Inoue K., Hirota S. et al. Changes in the incidence and prevalence of rheumatoid arthritis in Kamitonda, Wakayama, Japan 1965-1996 Ann Rheum Dis 1999; 58:751-756. 